\title{
Pathological torsional eye deviation during voluntary saccades: a violation of Listing's law
}

\author{
Christoph Helmchen, Stefan Glasauer, Ulrich Büttner
}

\begin{abstract}
Background-Under normal conditions, there are no torsional eye movements during voluntary saccades when the head is stationary (Listing's law).

Methods and results-Using dual search coils for three dimensional eye movement recordings, a patient is reported who had direction specific rapid deviations of torsional eye position (up to $10 \cdot 5^{\circ}$ ) during voluntary saccades followed by a slow exponential torsional drift after the end of the saccade ("blip") towards the initial torsional eye position. In the absence of spontaneous nystagmus, this transient torsion means a violation of Listing's law for voluntary saccades and was associated with a lesion involving the cerebellar vermis, its deep nuclei, and the dorsolateral medulla. Amplitudes of the blip were larger for ipsilesional (hypermetric) than contralesional (hypometric) horizontal saccades. For comparison transient torsion during and after saccades was also examined in six normal subjects. Using the same in vivo calibration, there were no blips larger than $1.2^{\circ}$ in any of them. Conclusion-Transient torsion with large amplitudes can be clinically seen on bedside examination and might thus be a new clinical sign in the diagnosis of saccadic disorders.
\end{abstract}

(F Neurol Neurosurg Psychiatry 1997;62:253-260)

Keywords: Listing's law; blips; torsional eye deviations; voluntary saccades

Torsional nystagmus ${ }^{12}$ and static torsion of the eyes ${ }^{3}$ are known clinical disorders of torsional eye movements and are of topodiagnostic value in patients with brainstem lesions. We present a new clinical sign of pathological torsional saccadic eye movements (blips) in the absence of torsional nystagmus and static ocular torsion, that might help in the diagnosis of saccadic disorders.

The eyes have three degrees of rotational freedom. Roughly, with respect to the head, the eyes can rotate around an earth vertical axis, a horizontal interaural axis, and the (torsional) axis of straight ahead gaze. Due to the non-commutative properties of eye rotations their sequence has to be taken into account to determine the final eye position after eye movements. According to Donders, the torsional component of the final eye position is determined by the horizontal and vertical component of the eye movement. There is one single eye position, called primary position, from which any other horizontal or vertical eye position can be reached through rotation about an axis which lies in a head fixed plane (Listing's plane) ${ }^{45}$ Thus with respect to this primary position, eye movements with the head stationary are restrained to two degrees of freedom of movement-horizontal and vertical. This restriction of eye movements was initially found by Helmholtz ${ }^{6}$ and is known as Listing's law. According to this law there should be no torsional eye movements during voluntary saccades-that is, the final eye orientation is restricted to orientations (axes of rotations) that can be reached from the primary position by rotation about an axis in Listing's plane.

Three dimensional eye position can be described in different coordinate systems. Commonly, rotation vectors ${ }^{7}$ or quaternions ${ }^{8}$ in a head fixed coordinate system are used because this representation helps to easily determine if axes of rotations indeed come to lie in Listing's plane. The components of a rotation vector describe the direction of the axis of rotation and the angle of rotation relative to a reference position. We used a special type of rotation vectors-quaternions. ${ }^{8}$ If the eye is inappropriately torted for any given eye position in space it constitutes a violation of Listing's law. Graphically, these quaternions show whether torsional eye positions lie out of Listing's plane (out of the plane of zero torsion) and hence violate Listing's law-that is, they indicate if there are torsional eye rotations during saccades. All eye positions in this study were transformed into quaternions relative to primary position after calculating Listing's plane. ${ }^{9}$ Primary position, which is the origin of the coordinate system using rotation vectors, is, however, not identical with the midposition or the gaze straight ahead position which roughly equals the centre of the oculomotor range. ${ }^{5}$

There are contradictory reports about the validity of Listing's law with respect to saccades in normal subjects. ${ }^{510-13}$ Recently, transient torsional eye displacements (blips) have been shown in normal subjects during voluntary saccades reaching torsional amplitudes up to $0.5-1^{\circ 11}{ }^{14}$ and $1-2^{\circ} .{ }^{13}$ However, it is not known how transient torsion evolves and when it becomes pathological. Moreover, the magnitude of transient torsion during saccadeshereafter called blips (for definition see methods)-has become crucial to test the 
validity of different models of the oculomotor velocity to position transformation. ${ }^{14}$

Using the search coil technique for recording three dimensional eye movements, we report a violation of Listing's law in a patient who showed a rapid deviation of torsional eye position during voluntary saccades followed by a slow exponential torsional drift after the saccade end towards the initial torsional eye position. There was no torsional or any other nystagmus in the light. This unusual oculomotor sign was associated with a unilateral lesion involving the cerebellar vermis, its deep nuclei, and the dorsolateral medulla.

\section{Patient and methods}

The 44 year old man had a history of sudden onset of severe vertigo, vomiting, coordination problems in his left arm and leg, gait unsteadiness, and a left facial numbness. Two weeks before these symptoms he had had a moderate whiplash trauma with subsequent headache lasting days but without other neurological symptoms. Otherwise, his medical history was unremarkable. There was a left cerebellar ataxia but no spontaneous nystagmus was noticed. Brain CT and MRI disclosed an infarction of the medical branch of the left posterior inferior cerebellar artery. ${ }^{15}$ The lesion involved posterior parts of the cerebellar hemisphere, the posterior vermis, nodulus, medial, and caudal parts of the deep cerebellar nuclei, including the fastigial nucleus, and the dorsolateral medulla oblongata (fig 1). The middle cerebellar peduncle was spared. Medially, immediately adjacent to the infarction, there was a small arachnoid cyst with a

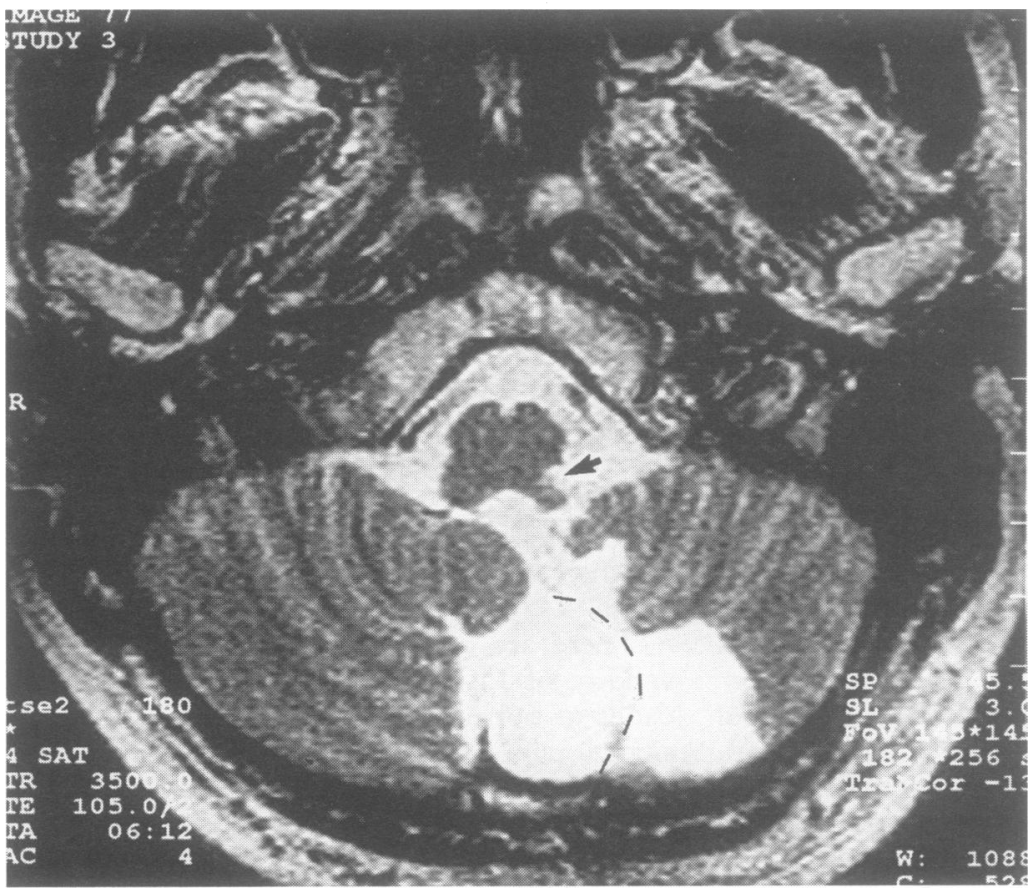

Figure 1 MRI in axial section (T2 weighted image) through the cerebellum and brainstem. The lateral border of the arachnoid cyst is indicated by the curved dashed line. The left posterior inferior cerebellar artery infarction involves parts of the posterior cerebellar hemisphere, the posterior vermis, the nodulus, the deep cerebellar nuclei, and the left lateral medulla oblongata (arrow). diameter of $2 \mathrm{~cm}$. Apart from that, MRI was normal.

Except for vertigo during head movements all symptoms remitted within months. One year after the infarction he came to our outpatient unit to ask for specific treatment of the persisting vertigo. There was no vertigo or spontaneous nystagmus in the light when the head was kept stationary. During large horizontal saccades and during vertical and horizontal smooth pursuit eye movements-which were saccadic at higher frequencies-some quick torsional deviation of both eyes was noticed by clinical examination. On head shaking movements in the horizontal plane or head positioning towards the left side there was a torsional nystagmus (beating in a negative torsional direction, counterclockwise-for definition see below) with a downward and small leftward quick component. During this nystagmus the patient noticed rotatory vertigo and unsteadiness. On serial positioning manoeuvres this nystagmus did not fatigue. Tilting the patient's head to the right did not evoke nystagmus or vertigo. The remaining general neurological examination was normal. In particular, there were no signs of ataxia or signs of the involvement of lower cranial nerves.

Angiography disclosed an occlusion of the left vertebral artery. In the distal part of the vertebral artery there was some supply from the ascending cervical artery; the right vertebral artery was hypoplastic. Within this limited vascular visualisation, there were no signs of vascular dissection, thrombosis, or an embolus. Cardioembolic causes were excluded by ultrasonography. Because of the poor vertebral artery blood supply anticoagulation therapy (coumadin) was initiated to prevent further infarction. An attempt was made to treat the vertigo elicited by head movement with up to $40 \mathrm{mg}$ baclofen four times daily. After six weeks of therapy without subjective or objective improvement baclofen was discontinued.

CONVENTION FOR DESCRIBING TORSIONAL EYE MOVEMENTS AND BLIPS

As mentioned above we used rotation vectors-quaternions-to describe eye rotations. ${ }^{8}$ Thus all eye positions were transformed into quaternions after calculating Listing's plane, ${ }^{9}$ the plane in which all axes of rotations come to lie relative to primary position.

Direction of torsional nystagmus and blips is described by the fast phase and defined from the subject's point of view according to the right hand rule. Rotation of the upper poles to the left - that is, intorsion of the right eye and extorsion of the left eye-is defined as negative torsion. Thus positive torsion equals clockwise torsion from the subjects' point of view, negative torsion counterclockwise rotation. Accordingly, upward rotation (around the earth's horizontal interaural axis) and rightward rotation (around an earth vertical axis) have a negative polarity. Blips are referred to as the torsional deviation during and after a saccade. ${ }^{14}$ They consist of a fast torsional deviation during the saccade and a slow exponen- 
tially decaying drift back to the presaccadic torsional eye position. Blip amplitude was computed as the difference between torsional eye position at onset of the saccade and maximal torsional deviation. According to Listing's law, this difference should always be zero. The time constant of the slow drift was calculated by fitting an exponential fit through the data points of the slow drift. ${ }^{16}$

METHODS OF EYE MOVEMENT RECORDINGS After the patient and normal subjects had given informed consent a scleral coil was placed in the left eye under topical anaesthesia. A three dimensional scleral induction coil (Skalar) was used for recording horizontal, vertical, and torsional eye movements in a dual search coil system with two orthogonal magnetic fields. Calibration was performed in vivo by an iteration algorithm depending only on the fixation of the straight ahead position and spontaneous eye movements (Bartl et al ${ }^{17}$; see also for description of the coil technique). Eye movement signals were digitised with a 12 bit $\mathrm{AD}$ converter (DAP 1200) at a sampling rate of $200 \mathrm{~Hz}$. The patient and normal subjects were asked to look at defined target locations presented by light emitting diodes on a tangent screen within a distance of $1 \mathrm{~m}$ from the subject's eye. Spontaneous and visually guided horizontal and vertical saccades $\left( \pm 10^{\circ}, \pm 20^{\circ}\right)$ were performed in random order. Smooth pursuit was elicited sinusoidally with amplitudes of $10^{\circ}$ and $20^{\circ}$ in the vertical and horizontal plane at $0.13,0.25$, and $0.4 \mathrm{~Hz}$. Pursuit performance (gain) was calculated by the ratio of eye amplitude (velocity) to stimulus amplitude (velocity) after omitting saccades. The head was kept stationary with a head holding device.

For caloric irrigation and testing the vestibulo-ocular reflex conventional electrooculography was added. ${ }^{18}$ Fundus photography and the determination of the subjective visual vertical were performed as described previously (for normative values in control subjects $\left.\mathrm{see}^{3}{ }^{19}\right)$. In addition, a video device ( $50 \mathrm{~Hz}$ sampling frequency) was used to qualitatively document eye movements.
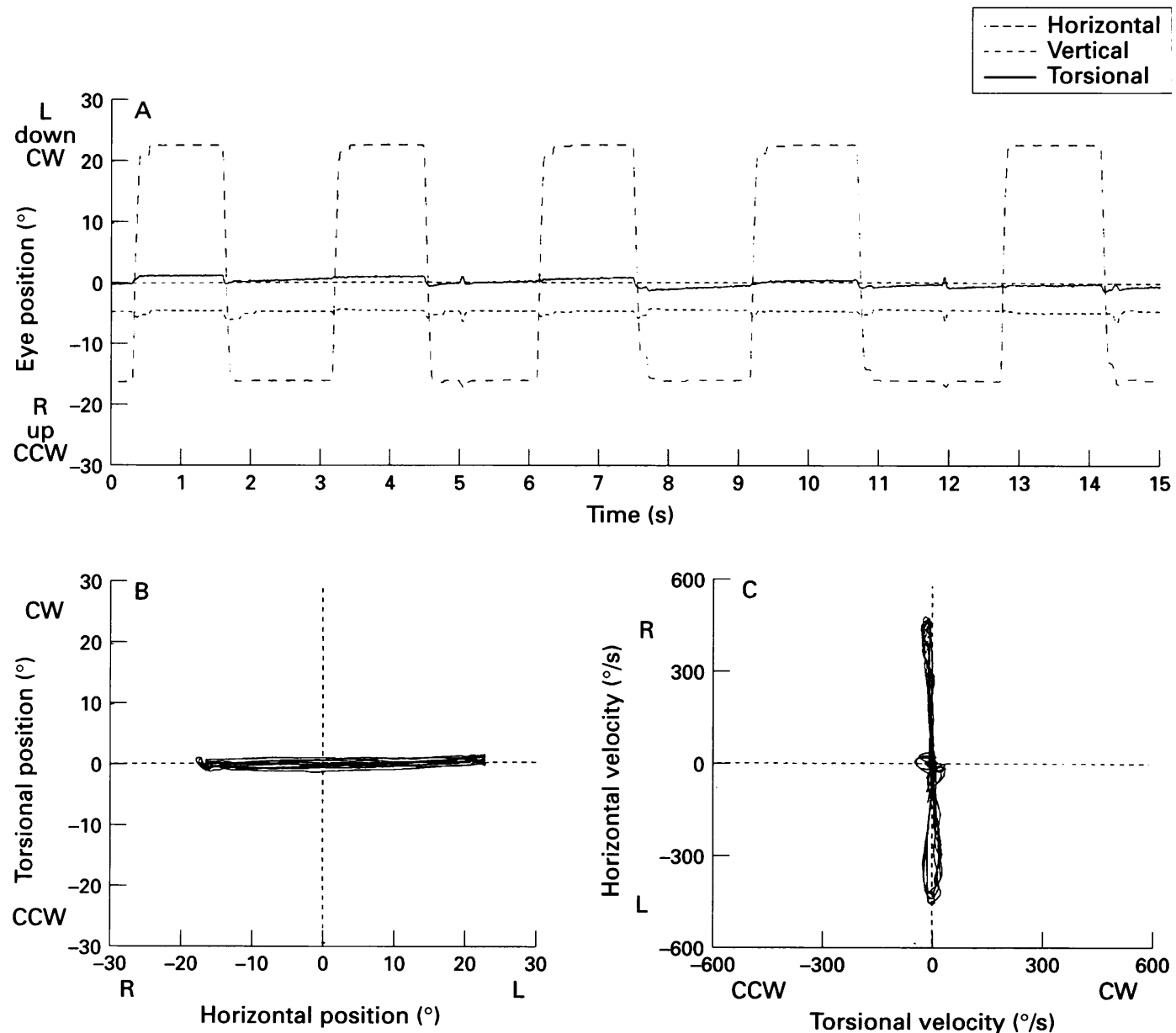

Figure 2 Search coil recording of horizontal, vertical, and torsional eye position of a normal subject. For better understanding, here and in the following figures, the amplitude is transformed into degrees ( ${ }^{\circ}$ ) and plotted as a function of time (s) using a quaternion coordinate system. Eye position is aligned to the zero torsion baseline, - that is, primary position (straight dashed horizontal line, see methods). Thus with respect to Listing's plane vertical eye position lies $5^{\circ}$ upwards from the straight ahead gaze position. Polarity of the ordinate has been adjusted according to the "right hand rule" (vertical positive = down; horizontal positive = leftward; torsional positive =CW). There is no or a very small torsional movement (blip, $<1^{\circ}$ ) during visually guided horizontal saccades. (A) $40^{\circ}$ across midposition. (B) Same saccades in a different projection, torsional eye position (ordinate) is plotted against horizontal eye position. There is no deviation from Listing's plane as indicated by the parallel saccade trajectories within a very small torsional eye position range. The trajectories of torsional (abscissa) and horizontal (ordinate) saccade velocity $(\%)$ in (C) illustrate torsional deviations from Listing's plane even better, which in this case are very small. According to the right hand rule angular velocity vectors are shown - that is, horizontal eye movements are displayed as velocity vectors in the yaw plane. $C W=$ positive (clockwise) torsion; $C C W=$ negative (counterclockwise) torsion. 


\section{Results}

EYE MOVEMENT RECORDINGS (NORMAL SUBJECTS):

Figure 2A shows a search coil recording of horizontal, vertical, and torsional eye position of a normal subject, displayed as quaternions being transformed into degrees for better understanding. As mentioned above, with respect to a particular reference position (primary position) all eye movements are restrained to two degrees of freedom of movement-horizontal and vertical. This becomes evident in a three dimensional coordinate system by different views on the eye positions (fig 2B). There are virtually no torsional deviations from the horizontal saccade trajectories (none or only very small blips). The thinner the Listing's plane, the better the saccades obey Listing's law. Standard deviation of the width of Listing's plane did not exceed $1 \cdot 1^{\circ}$ in our normal subjects (fig 2 ). Thus the widths of Listing's plane were compatible with other reports $^{13}$ and artifacts by calibration error are unlikely. ${ }^{17}$ Looking at torsional velocity trajectories provides an even better analysis of the deviations from Listing's plane (fig 2C). Here, we only describe oculomotor signs that were also tested on the patient and we refer to other recent studies for more detailed descriptions of blips. ${ }^{1314}$ Using the same paradigms as in the patient, the amplitude of torsional deviation (blip) during vertical and horizontal saccades did not exceed $1 \cdot 2^{\circ}$ in all six subjects, irrespective of direction and amplitude. For all six subjects, mean torsional blip amplitudes for $40^{\circ}$ saccades was on average $0.53 \pm 0.3$ (range $0 \cdot 1-0 \cdot 85)^{\circ}$. Time constants of the decay of the blip ranged from $80-250 \mathrm{~ms}$. Blip amplitude was not correlated or only weakly correlated with saccade amplitude (range of regression coefficient $r=0.35-0.65)$. Thus some horizontal saccades did not show blips at all, in particular during saccades across midposition (straight ahead gaze, fig 2). During horizontal saccades blips could change their direction, leftward saccades tended to show blips with a negative torsional direction (CCW); rightward saccades blips with a positive torsional direction (CW) respectively. Moreover, blip direction also changed with elevation of the eye-that is, blip direction on downward gaze was usually opposite to that of upward gaze. Thus saccade direction and elevation of eye position determined the magnitude and direction of the blip. ${ }^{13}$

\section{EYE MOVEMENT RECORDINGS (PATIENT) \\ Abnormalities during fixation}

In the light, on fixation of the straight ahead position (midposition) and up to $\pm 30^{\circ}$ horizontally and $\pm 20^{\circ}$ vertically there was no nystagmus. In darkness, there was a small spontaneous nystagmus (up to $3.5 \%$ s horizontal slow phase velocity) with a downward, leftward, and counterclockwise (negative torsional) quick component. It was completely suppressed on fixation in the light.

Abnormalities during saccades

Horizontal and vertical saccades $\left(20-40^{\circ}\right.$ amplitudes) had normal velocities. Horizontal saccades were hypermetric to the left (ipsilesional, gain average: $1.2 \pm 0.09$, range: 1.01-1.43) and hypometric to the right (contralesional, gain average: $0.85 \pm 0.07$, range: $0.5-0.96)$. Using the same paradigms as in the normal subjects, there were large transient torsional quick eye displacements during horizontal and vertical saccades (blips, fig $3 \mathrm{~A}, \mathrm{~B}$ ) that we did not find in normal subjects (see above). Quick torsional deviations usually started shortly after saccade onset and reached their maximum at the end of the horizontal or vertical saccade (fig 3A, B, C). Peak torsional deviation was always reached after the peak horizontal velocity during horizontal saccades (fig 3B, E). After the rapid uncalled for torsion there was a slow torsional drift back to the baseline torsional position exceeding the end of the horizontal and vertical component. The slow torsional drift showed an exponentially decreasing slow phase velocity in most instances. The time constant of the slow drift ranged from $250-310 \mathrm{~ms}\left(40^{\circ}\right.$ saccade amplitude). The rapid torsional component during saccades moves the eye out of Listing's plane whereas the torsional slow drift brings the eye back to the plane (fig 3C-E), a phenomenon not seen in (or present to a much lesser degree in) normal subjects. Figure $3 E$ shows that torsional velocity still increases when horizontal velocity has reached its maximum. In the patient, blips were always beating in a negative torsional direction, irrespective of the horizontal or vertical eye position. Mean torsional velocity for $5^{\circ}$ amplitudes was $95 \%$. Blip amplitude could be as large as $10.5^{\circ}$ but could vary for the same saccade amplitude up to $6^{\circ}$ (fig 4). However, on average it clearly depended on the direction of the horizontal saccades. Leftward saccades were associated with larger torsional (blip) amplitudes than rightward saccades, particularly for larger saccades (fig 4). Blip amplitudes during hypermetric (leftward) saccades were significantly larger than during hypometric (rightward) saccades (for example, blip amplitude for $10^{\circ}$ saccades $1.37 \pm 1.1$ to the right and $2.5 \pm 1.2$ to the left, $P<0.05$, fig 4). Blip amplitude was correlated with leftward (hypermetric) $(r=$ $0.88, \mathrm{P}<0.0001$ ) but only weakly correlated with rightward (hypometric) saccade amplitudes $(r=0.33, \mathrm{P}<0.03$, fig 4). Accordingly, vertical saccades also showed blips with a negative torsional direction (CCW).

\section{Abnormalities during smooth pursuit eye movements}

Horizontal smooth pursuit eye movements were normal at low frequencies $(0.25 \mathrm{~Hz}$ : gain $0.90 \pm 0.06)$ and mildly saccadic at higher frequencies $(0.4 \mathrm{~Hz}$ : gain $0.78 \pm 0.04$ to the left, $0.61 \pm 0.11$ to the right). Vertical smooth pursuit eye movements were mildly saccadic at $0.4 \mathrm{~Hz}$ (up: $0.61 \pm 0.11$, down: $0.55 \pm 0.09$, normal to age matched subjects). During vertical and horizontal smooth pursuit eye movements torsional deviations (always negative torsional quick deviations) only occurred when there were saccades in the vertical or 
Figure 3 Search coil recording of transient torsion (blip) during and after horizontal saccades across the midposition (eye position in straight ahead gaze) in the patient. ( $A$, B) Time course of one blip with single trajectories of vertical, horizontal, and torsional eye position $A$, in $\left.{ }^{\circ}\right)$ and velocities $B$, in $(\mathrm{o} / \mathrm{s})$ is shown. Primary position is indicated by the dotted straight horizontal line at $0^{\circ}$. (A) Torsional rapid deviation component reaches its maximum at the end of the horizontal eye position component followed by a long slow drift back to primary position. (B) Peak velocity of the quick deviation of the torsional component lags behind that of the horizontal velocity component. Vertical dashed line indicates peak velocity of the horizontal eye component. For better comparison, different scales have been taken left ordinate for horizontal/ vertical velocity and right ordinate for torsional velocity). (C) Horizontal, vertical, and torsional eye position (ordinate) of the patient is shown for 10 s. Blip consists of a quick torsional movement out of the zero torsion plane (horizontal straight line) and is followed by a slow drift back to this plane. Gaze straight ahead is not equal to zero horizontal eye position (for example,

horizontal eye position in this example lies about $4^{\circ}$ to the left). (D) Torsional (ordinate) against

horizontal eye position

(abscissa). During and

after the horizontal saccade (arrow) there is torsional deviation from Listing's

plane with a slow drift

back to the plane of zero torsion (Listing's plane) (E) Torsional velocity $(\% / s$, abscissa) is plotted $v$ horizontal velocity (according to the right hand rule angular velocity vectors are shown - that is, horizontal eye movements are displayed as velocity vectors in the yaw plane). Note the curved saccade trajectories out of the zero torsion plane and their slow back drift as indicated by the arrows $(\leftarrow, D, E)$ both of which are larger for leftward saccades.

Leftward saccades have larger blip amplitudes than rightward saccades.
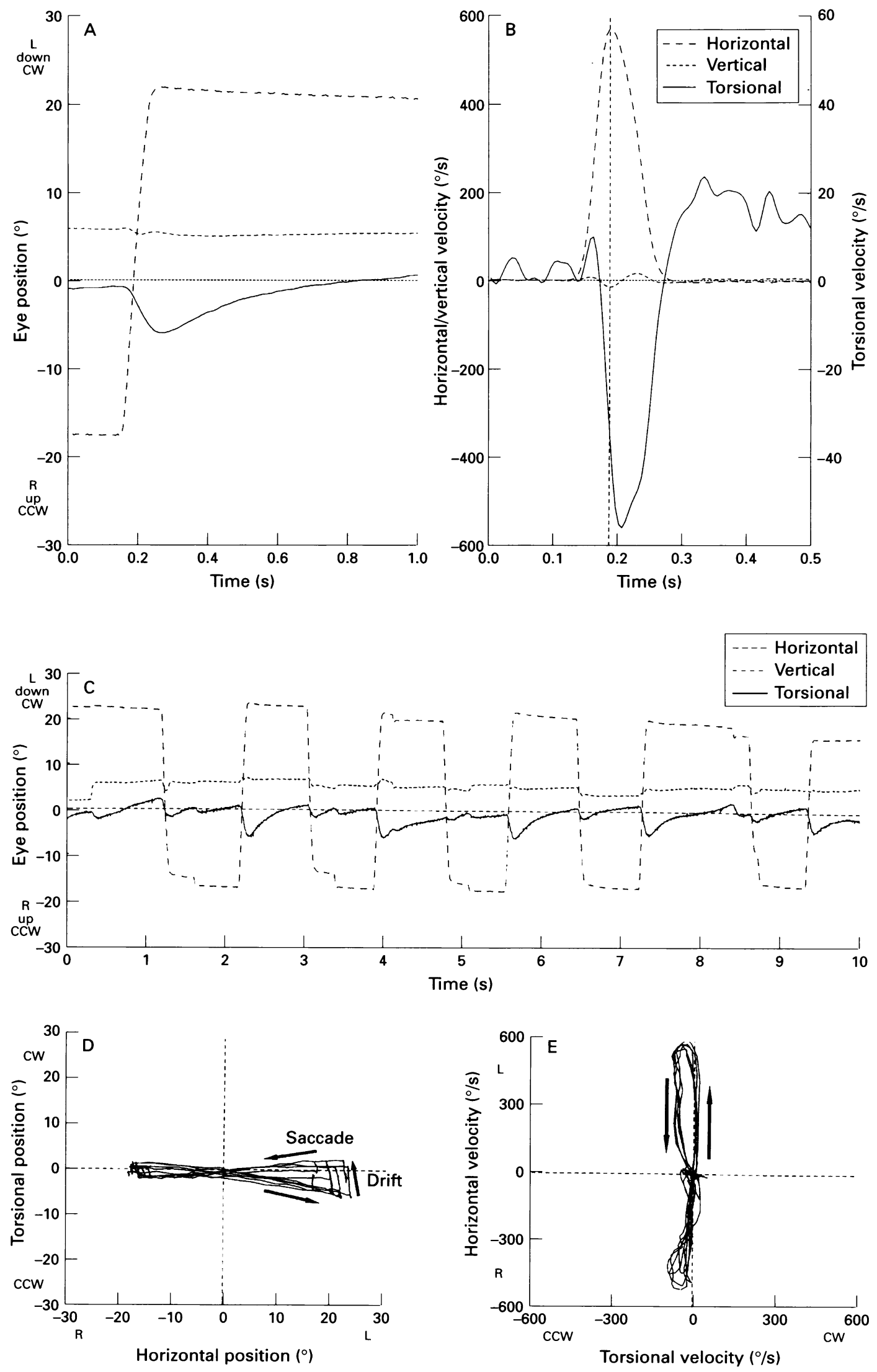

horizontal eye pursuit component. Torsional slow phase eye velocity (slow return after saccade end) was not related to vertical smooth pursuit velocity. Torsional displacement during saccadic vertical pursuit at higher frequencies $(0.4 \mathrm{~Hz})$ did not change its direction, but downward pursuit at higher frequencies was slightly more saccadic than upward pursuit and showed larger blips than upward pursuit.

Additional electro-oculographic recordings in the patient disclosed an asymmetric horizontal vestibulo-ocular reflex gain in the dark (leftward, ipsilesional gain: $0.9 \pm 0.03$; rightward, contralesional gain: $0.55 \pm 0.16)$. Caloric irrigation (warm, $44^{\circ}$; cold $30^{\circ}$ ) excluded a peripheral vestibular disorder. 
Figure 4 Blip amplitudes (ordinate) as a function of leftward (O, $n=25)$ and rightward ( $\square, n=$ 45) horizontal saccades. Blips with leftward saccades always have a negative torsional component and larger amplitudes (up to $10.5^{\circ}$ ) Some rightward saccades have zero blip amplitudes or small $\left(<1^{\circ}\right)$ positive torsional deviations.

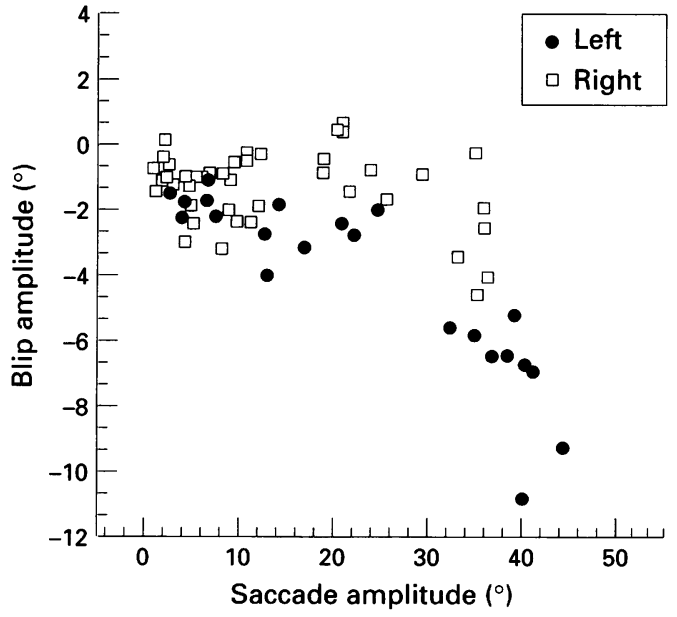

FUNDOSCOPY AND SUBJECTIVE VISUAL VERTICAL

Serial fundus photography of the patient disclosed no signs of pathological tonic ocular torsion $\left(6-8^{\circ}\right.$ excyclotropia of the right eye and $4-7^{\circ}$ excyclotropia of the left eye). Similarly, the subjective visual vertical was normal under static $\left(-2 \cdot 0^{\circ}\right)$ and dynamic conditions (positive torsional direction $+16^{\circ}$, negative torsional direction $\left.-17 \cdot 8^{\circ}\right) .^{3}$

\section{Discussion}

The main finding of this study was the presence of pathological torsional eye deviations during voluntary saccades with the head stationary in a patient who had no spontaneous nystagmus in the light. This indicates a violation of Listing's law. Torsional eye deviations during saccades have been called blips. ${ }^{13} 14$ This term exclusively refers to torsion during and after saccades and is more specific than other terms-for example, pathological torsion.

For clinical purposes, we have shown that the unusual magnitude of the blip - which has not been reported before-reflects a pathological sign compared with normal persons. We used an in vivo calibration ${ }^{17}$ and found blips in normal subjects that did not exceed $1 \cdot 2^{\circ}$ amplitude. Thus none of our normal subjects showed blips of the magnitude we saw in our patient. Slippage of the coil on the eye seems unlikely because neither we nor others ${ }^{13}$ found torsional drifts of this magnitude in normal subjects; repetition of coil recordings of the same normal subject yielded the same results. Moreover, we have shown that torsional saccade amplitude was direction specific and correlated with the amount of saccadic dysmetria indicating neuronal implementation of this unusual oculomotor sign. This direction specificity of blip amplitude in our patient also suggests a specific, reproducible effect.

Inappropriate cross coupling of vertical and horizontal or torsional saccades has been described in Wallenberg's syndrome, leading to saccadic "lateropulsion" 20 or "torsional pulsion".2 "Torsional pulsion", as suggested by Morrow and Sharpe, implies an unwanted torsional deviation during voluntary saccades.
Thus torsional pulsion and blips might be a related phenomenon. However, there are some differences of torsional eye movements in the latter study compared with blips in our patient. All patients in that study had spontaneous torsional nystagmus which decreased or disappeared in darkness - that is, there was a constant drift of torsional eye position on fixation, which was only interrupted by quick phases of nystagmus. In our patient, however, torsional eye position remained stable even throughout longer periods of fixation. In two of their three patients there were-in addition to the torsional nystagmus - transient torsional deviations during vertical and horizontal saccades. ${ }^{2}$ Torsional nystagmus was beating to the contralesional brainstem side $^{2}$ whereas blips in our patient showed a quick phase to the side of the lesion. It should be stressed that during nystagmus the eye deviates from a certain position and is brought back by the saccade. In our patient torsional deviation clearly occurs with a fast movement which is reset by a slow exponential drift. Using rotation vectors-quaternions-helps to show that the fast torsional deviation of the blip pushes the eye out of Listing's plane and the slow exponential drift brings it back towards the plane.

If transient torsion during saccades was caused by the same mechanism underlying saccadic dysmetria blip amplitude would be expected to be correlated with the amount of saccadic dysmetria. In our patient ipsilesional (leftward) saccades were hypermetric and correlated with blip amplitude (fig 4). As visually guided torsional eye movements are not possible we cannot look for "torsional saccadic dysmetria". Torsional deviation during horizontal saccades may reflect dysmetria in the roll plane. Because the eye drifts back to Listing's plane as the "torsional target position" we need to look for this reference system to evaluate "torsional dysmetria". In this sense, Listing's plane provides a reference system of metric torsional eye movements which, in addition, is nearly independent of the cooperation of the patient.

Inappropriate coupling of torsional and pursuit signals has recently been found in patients who had no transient torsion (blips) during saccades. ${ }^{21}$ This torsional nystagmus has been explained by asymmetric pursuit signals due to an imbalance of oppositely directed torsional components which are usually cancelled to generate pure vertical pursuit eye movement without torsion. However, we only found torsional deviations (blips) associated with saccades and never in relation to slow eye movements such as smooth pursuit or slow phases of vestibular nystagmus. Torsional slow phase velocity was not related to the velocity of vertical smooth pursuit. We only found torsional deviations during vertical and horizontal pursuit when pursuit - at higher frequencies was interrupted by saccades. In our patient, torsional deviations did not change their directions during upward or downward pursuit eye movements although they were much smaller during upward pursuit. Thus the torsional deficits in our patient were not related to pur- 
suit signals but constitute a saccadic disorder. Finally, unlike the results of FitzGibbon et al ${ }^{21}$ the middle cerebellar peduncle was clearly not lesioned in our patient.

Taking all lines of evidence together, our data suggest that the pathological blips in our patient reflect "torsional saccadic dysmetria" and not the fast resetting response to a (pathological) slow phase torsional velocity signal, the second being the normal cause for eliciting a torsional saccade component. It is in agreement with this view that in our patient the torsional component drifts back after its maximal deviation with a time constant close to that found after mechanical torsional displacements. ${ }^{16}$

At present it remains speculative whether blips in our patient are caused by the lateral medullary or the cerebellar lesion. There were no additional clinical brainstem signs indicative of Wallenberg's syndrome. Pathological static torsion (fundoscopy) or deviation of the subjective visual vertical were absent, and these are often seen ( $94 \%$ of patients) after dorsolateral medullary lesions. ${ }^{3}$ Nevertheless the medullary lesion in our patient could explain the saccadic disorders by virtue of a disruption of climbing fibres. It has previously been shown that saccadic dysmetria in Wallenberg's syndrome is probably caused by a functional lesion of the deep cerebellar nuclei ${ }^{18}$ - that is, the oculomotor vermis and the fastigial nucleus. These structures, which receive afferent climbing fibre input, are also involved in the infarction of our patient.

\section{THEORETICAL IMPLICATIONS}

The magnitude of transient torsion during saccades has recently become a crucial argument in the testing of the validity of two models concerning the neural representation of the velocity to position transformation. The question arises how the three dimensional velocity to position integrator contributes to this transient torsion ${ }^{13}$ - for example, does the neural integrator try to obey Listing's law only at equilibrium or at all times but fails due to mismatches between the premotor signal and the oculomotor plant dynamics?

The small blip amplitude in normal subjects was taken as evidence against the SchnabolkRaphan model, ${ }^{22}$ predicting torsional deviations of up to $7^{\circ}$, and was more compatible with the quaternion model of velocity to position transformation, ${ }^{14}$ predicting no blips at all. It is of importance, that both models use identical values for passive torques. The Schnabolk-Raphan model suggests that the eye is moved by torques of the extraocular muscles, representing commutative vectors, and thus a neural representation of commutative vector transformation is sufficient to generate eye movements. Thus using torque vectors the model preserves Listing's law by the elastic properties of the oculomotor plant. The quaternion model ${ }^{14}$ instead is based on a non-commutative process of oculomotor velocity to position transformation. Blips of small amplitudes would only occur if torsional burst amplitude is not appropriate.
POSSIBLE MECHANISMS OF BLIP GENERATION If there is a neural representation of the torsional equilibrium eye positions somewhere in the brainstem or cerebellum ("Donders box"4), a failure of this structure would imply a loss of control that usually prevents and corrects torsional deviations during saccades. Consequently, pathological torsion (blips) would occur. However, torsional deviation could also be actively produced ("torsional saccadic dysmetria") if there is a mismatch between the premotor saccadic signal (pathological saccadic pulse) and the oculomotor plant dynamics. The rapid torsional deviations would interfere with the saccade command, thus leading to torsional deviations. Theoretically, it can also be postulated that the non-commutative velocity to position integrator $^{14}$ lost its eye position feedback signal, which transforms it into a commutative integrator closely resembling the SchnabolkRaphan model, thus producing substantial blip amplitudes. By contrast, to change the blip amplitude from $1-2^{\circ}$ (normal subject) to 5-10 (patient), the Schnabolk-Raphan model would make a substantial change of burst parameters necessary, which can probably not be explained by the lesioned neuronal structures in our patient.

Finally, what brings the eye back to Listing's plane? Interestingly, time constants of the drift back to the torsional position at saccade onset were similar to those found for drifts after mechanical torsional displacements. ${ }^{16}$ Thus in this respect the drift would just reflect the elastic properties of the oculomotor plant.

\section{Conclusion}

We have provided evidence for pathological torsional deviations during and after voluntary saccades (blips). This indicates a violation of Listing's law for saccades. In the absence of torsional nystagmus pathological torsional deviations during saccades (blips) might be a new helpful clinical sign for the diagnosis of torsional eye movement disorders, particularly as this sign can be clinically seen. The direction specificity of blips in our patient being closely aligned to the pattern of horizontal saccadic dysmetria could suggest similar underlying pathomechanisms providing evidence for "torsional saccadic dysmetria". For clinical use, blips can be best seen during large horizontal saccades.

This work was supported by the Deutsche Forschungsgemeinschaft (DFG)

1 Lopez L, Bronstein AM, Gresty MA, Rudge P, DuBoulay E. Torsional nystagmus. A neuro-otological and MRI study of thirty-five cases. Brain 1992;115:1107-24.

2 Morrow MJ, Sharpe JA. Torsional nystagmus in the lateral medullary syndrome. Ann Neurol 1988;24:390-8.

3 Dieterich M, Brandt Th. Ocular torsion and tilt of subjective visual vertical are sensitive brainstem signs. Ann Neurol 1993;33:292-9.

4 Hepp K. Listing's law and all that. Current Opinion in Neurobiology 1994;4:862-8.

5 Tweed D, Vilis T. Geometric relations of eye position and velocity vectors during saccades. Vision Res 1990;30: velocity

6 von Helmholtz H. Handbuch der Physiologischen Optik. Leipzig: Voss, 1867. 
7 Haustein W. Considerations on Listing's law and the primary position by means of a matrix description of eye position control. Biol Cybern 1989;60:411-20.

8 Tweed D, Vilis T. Implications of rotational kinematics for the oculomotor system in three dimensions Neurophysiol 1987.58:832-49.

9 Tweed D, Cadera W, Vilis T. Computing three-dimensional eye position quaternions and eye velocity from sional eye position quaternions and eye ve
search coil signals. Vision Res 1990;30:97-110.

10 Minken AH, van Opstal AJ, van Gisbergen JM. Threedimensional analysis of strongly curved saccades elicited dimensional analysis of strongly curved saccades elicited

11 Enright JT. The aftermath of horizontal saccades: saccadic retraction and cyclotorsion. Vision Res 1986;26:1807-14

12 Ferman L, Collewijn H, Van der Berg AV. A direct test of Listing's law-II. Human ocular torsion measured under dynamic conditions. Vision Res 1987;27:939-51.

13 Straumann D, Zee DS, Solomon D, Lasker AG, Roberts DC. Transient torsion during and after saccades. Vision Res 1995;35:3321-34.

14 Tweed D, Misslisch H, Fetter M. Testing models of the oculomotor velocity-to-position transformation. $\mathcal{F}$ Neurophysiol 1994;72:1425-9.

15 Amarenco P, Roullet E, Hommel M, Chaine P, Marteau R. Infarction of the territory of the medial branch of the pos- terior inferior cerebellar artery. $\mathcal{F}$ Neurol Neurosurg Psychiatry 1990;53:731-5.

16 Seidman SH, Leigh RJ, Tomsak RL, Grant MP, Dell'Osso LF. Dynamic properties of the human vestibulo-ocular reflex during head rotations in roll. Vision Res 1995; 35:679-89.

17 Bartl K, Siebold C, Glasauer S, Helmchen C, Büttner U. A simplified calibration method for 3D eye movement simplified calibration method for $3 \mathrm{D}$ eye movement
recordings using search coils. Vision Res 1996;36: recordings

18 Helmchen $\mathrm{Ch}$, Straube A, Büttner U. Saccadic lateropulsion in Wallenberg's syndrome may be caused by a functional lesion of the deep cerebellar nucleus. $\mathcal{F}$ Neurol 1994;241:421-6.

19 Dieterich $M$, Brandt Th. Wallenberg's syndrome: Lateropulsion, cyclorotation, and subjective visual verti$\mathrm{cal}$ in thirty-six patients. Ann Neurol 1992;31:399-408.

20 Kommerell G, Hoyt WF. Lateropulsion of saccadic eye movements. Arch Neurol 1973;28:313-8.

21 FitzGibbon EJ, Calvert PC, Dieterich M, Brandt T, Zee DS. Torsional nystagmus during vertical pursuit. $\mathcal{F}$ Neuroophthalmol 1996;16:79-90.

22 Schnabolk C, Raphan T. Modeling three-dimensional velocity-to-position transformation in oculomotor control. $\mathcal{F}$ Neurophysiol 1994;71:623-38. 